\title{
Mitochondrial Oxidative Stress and Heart Failure
}

\author{
Hiroyuki Tsutsui $^{1}$
}

\begin{abstract}
Recent experimental and clinical studies have suggested that oxidative stress is enhanced in heart failure. Chronic increases in oxygen radical production in the mitochondria can lead to a catastrophic cycle of mitochondrial DNA (mtDNA) damage as well as functional decline, further oxygen radical generation, and cellular injury. Reactive oxygen species induce myocyte hypertrophy, apoptosis, and interstitial fibrosis by activating matrix metalloproteinases. These cellular events play an important role in the development and progression of maladaptive cardiac remodeling and failure. Overexpression of mitochondrial transcription factor A (TFAM) could ameliorate the decline in mtDNA copy number and preserve it at a normal level in failing hearts. Consistent with alterations in mtDNA, the decrease in oxidative capacities was also prevented. Therefore, the activation of TFAM expression could ameliorate the pathophysiological processes seen in myocardial failure. Inhibition of mitochondrial oxidative stress and DNA damage could be the most effective and novel treatment strategies for heart failure.
\end{abstract}

Key words: heart failure, remodeling, oxidative stress, mitochondria, DNA

(DOI: 10.2169/internalmedicine.45.1765)

\section{Introduction}

Heart failure is a leading cause of morbidity and mortality in industrialized countries (1). It is also a growing public health problem, mainly because of aging of the population and the increase in the prevalence of heart failure in the elderly. Previous basic, clinical, and population sciences have advanced the modern treatment of heart failure. Despite extensive studies, the fundamental mechanisms responsible for the development and progression of left ventricular (LV) failure have not yet been fully elucidated.

Reactive oxygen species (ROS) such as superoxide anions $\left(\cdot \mathrm{O}_{2}{ }^{-}\right)$and hydroxy radicals $(\cdot \mathrm{OH})$ cause the oxidation of membrane phospholipids, proteins, and DNAs (2) and have been implicated in a wide range of pathological conditions including ischemia-reperfusion injury, neurodegenerative diseases, and aging. Under physiological conditions, their toxic effects can be prevented by such scavenging enzymes as superoxide dismutase (SOD), glutathione peroxidase (GSHPx), and catalase as well as by other non-enzymatic antioxidants. However, when the production of ROS becomes excessive, oxidative stress might have a harmful effect on the functional and structural integrity of biological tissue.
Recent experimental and clinical studies have suggested the generation of ROS to increase in heart failure (3-6). ROS cause contractile failure and structural damage in the myocardium. The importance of oxidative stress is increasingly emerging with respect to a pathophysiological mechanism of LV remodeling responsible for heart failure progression.

By using electron spin resonance (ESR) spectroscopy combined with the nitroxide radical, 4-hydroxy-2,2,6,6tetramethyl-piperidine-N-oxyl (hydroxy-TEMPO), a definitive and direct demonstration of enhanced generation of ROS was obtained in the failing myocardium (7). $\cdot_{2}$ is a primary radical that could lead to the formation of other ROS, such as $\mathrm{H}_{2} \mathrm{O}_{2}$ and $\cdot \mathrm{OH}$, in the failing myocardium. $\cdot \mathrm{OH}$ could arise from the electron exchange between $\cdot \mathrm{O}_{2} \cdot$ and $\mathrm{H}_{2} \mathrm{O}_{2}$ via the Harber-Weiss reaction. In addition, $\cdot \mathrm{OH}$ is also generated by the reduction of $\mathrm{H}_{2} \mathrm{O}_{2}$ in the presence of endogenous iron by means of the Fenton reaction. The generation of $\cdot \mathrm{OH}$ implies a pathophysiological significance of ROS in heart failure since $\cdot \mathrm{OH}$ radicals are the predominant oxidant species causing cellular injury.

The decreased antioxidant capacity could further aggravate the ROS accumulation in heart failure. However, the activities of SOD, catalase, and GSHPx were not decreased

\footnotetext{
${ }^{1}$ Department of Cardiovascular Medicine, Hokkaido University Graduate School of Medicine, Sapporo Correspondence to Hiroyuki Tsutsui, Department of Cardiovascular Medicine, Hokkaido University Graduate School of Medicine, Kita-15, Nishi-7, Kita-ku, Sapporo 060-8638
} 
in the failing hearts (8), indicating that oxidative stress in heart failure is primarily due to the enhancement of prooxidant generation rather than to the decline in antioxidant defense.

\section{Mitochondria as a source of oxidative stress}

The cellular sources of ROS generation within the heart include cardiac myocytes, endothelial cells, and neutrophils. Within cardiac myocytes, ROS can be produced by several mechanisms including mitochondrial electron transport, NADPH oxidase, and xanthine dehydrogenase/xanthine oxidase.

By using ESR spectroscopy with 5,5'-dimethyl-1pyrroline-N-oxide (DMPO) as a spin trap, the inhibition of electron transport at the sites of complex I and complex III in the normal submitochondrial particles resulted in a significant production of $\mathrm{O}_{2}^{-}(9)$. Mitochondria from heart failure produced more $\mathrm{O}_{2}^{-}$than normal mitochondria in the presence of $\mathrm{NADH}$, indicating that mitochondrial electron transport could be the predominant source of such ${ }^{-} \mathrm{O}_{2}^{-}$production. Furthermore, heart failure mitochondria were associated with a decrease in complex enzyme activity. Therefore, mitochondria are the predominant source of ROS in failing hearts, indicating a pathophysiological link between mitochondrial dysfunction and oxidative stress (10) as has been reported in other disease conditions including aging and neurodegenerative diseases.

\section{Consequences of oxidative stress in heart failure}

\section{Oxidative stress and mitochondrial DNA (mtDNA) damage}

ROS can damage mitochondrial macromolecules either at or near the site of their formation. Therefore, in addition to the role of mitochondria as a source of ROS, the mitochondria themselves can be damaged by ROS. The mtDNA could be a major target for ROS-mediated damage for several reasons. First, mitochondria do not have a complex chromatin organization consisting of histone proteins, which may serve as a protective barrier against ROS. Second, mtDNA has a limited repair activity against DNA damage. Third, a large part of $\cdot \mathrm{O}_{2}^{-}$, which is formed inside the mitochondria, can not pass through the membranes and, hence, ROS damage may be contained largely within the mitochondria. In fact, mtDNA accumulates significantly higher levels of the DNA oxidation product, 8-hydroxydeoxyguanosine, than nuclear DNA (11). As opposed to nuclear-encoded genes, mitochondrial-encoded gene expression is largely regulated by the copy number of mtDNA (12). Therefore, mitochondrial injury is reflected by mtDNA damage as well as by a decline in the mitochondrial RNA (mtRNA) transcripts, protein synthesis, and mitochondrial function (13, 14). We have shown that the increased generation of ROS is associated with mitochondrial damage and dysfunction in the failing heart, which were characterized by an increased lipid peroxidation in the mitochondria, a decreased mtDNA copy number, a decrease in the number of mtRNA transcripts, and a reduced oxidative capacity due to low complex enzyme activities (15). Chronic increases in ROS production are associated with mitochondrial damage and dysfunction which thus can lead to a catastrophic cycle of mitochondrial functional decline, further ROS generation, and cellular injury. MtDNA defects may thus play an important role in the development and progression of myocardial remodeling and failure.

\section{Oxidative stress and myocardial damage}

ROS have direct effects on cellular structure and function and may be integral signaling molecules in myocardial remodeling and failure. ROS result in a phenotype characterized by hypertrophy and apoptosis in isolated cardiac myocytes (16). ROS have also been shown to activate matrix metalloproteinase (MMP) in cardiac fibroblasts (17). Myocardial MMP activity is increased in the failing hearts (18, 19). Further, an MMP inhibitor has been shown to limit early LV dilatation in a murine model of MI (20). We have shown the significant improvement in the survival after MI in MMP-2 knockout mice, which was mainly attributable to the inhibition of early cardiac rupture and the development of subsequent LV dysfunction (21). Because MMP can be activated by ROS (22), one proposed mechanism of LV remodeling is the activation of MMPs secondary to increased ROS production. Sustained MMP activation might influence the structural properties of the myocardium by providing an abnormal extracellular environment with which the myocytes interact. We have demonstrated that $\cdot \mathrm{OH}$ scavenger, dimethylthiourea, inhibits the activation of MMP-2 in association with the development of LV remodeling and failure (23). These data raise the interesting possibility that increased ROS after MI can be a stimulus for myocardial MMP activation, which might play an important role in the development of heart failure.

\section{Amelioration of mitochondrial oxidative stress, mtDNA damage, and heart failure}

\section{GSHPx}

The first defense mechanism against ROS-mediated cardiac injury comprises several antioxidant enzymes including SOD, catalase, and GSHPx. Among these antioxidants, GSHPx is an important enzyme that performs several vital functions. GSHPx is a key antioxidant which catalyses the reduction of $\mathrm{H}_{2} \mathrm{O}_{2}$ and hydroperoxides. It not only scavenges $\mathrm{H}_{2} \mathrm{O}_{2}$ but also prevents the formation of other more toxic radicals such as $\cdot \mathrm{OH}$. GSHPx possesses a higher affinity for $\mathrm{H}_{2} \mathrm{O}_{2}$ than catalase. Further, it is present in relatively high amounts within the heart especially in the cytosolic and mitochondrial compartments (24). These lines of evidence im- 
ply the primary importance of GSHPx as a defense mechanism within the heart compared to catalase. Moreover, GSHPx is expected to exert greater protective effects against oxidative damage than SOD because greater dismutation of $\cdot \mathrm{O}_{2}^{-}$by SOD may result in an increase of $\mathrm{H}_{2} \mathrm{O}_{2}$. Therefore, compared with SOD or catalase, GSHPx is thought to be more effective in protecting cells, tissues, and organs against oxidative damage (25).

GSHPx overexpression inhibits the development of LV remodeling and failure after myocardial infarction (MI), which might contribute to the improved survival (26). These findings not only extended the previous observation that employed antioxidants, but also revealed the major role of ROS in the pathophysiology of post-MI remodeling. These effects were associated with the attenuation of myocyte hypertrophy, apoptosis, and interstitial fibrosis (26). Therefore, therapies designed to interfere with oxidative stress by using GSHPx could be beneficial to prevent heart failure.

\section{Peroxiredoxin-3 (Prx-3)}

We have recently demonstrated that the overexpression of a mitochondrial antioxidant, Prx-3, a member of peroxiredoxin family that can scavenge $\mathrm{H}_{2} \mathrm{O}_{2}$ in cooperation with thiol and peroxynitrite, protects the heart against post-MI remodeling and failure in mice. It reduces LV cavity dilatation and dysfunction as well as myocyte hypertrophy, interstitial fibrosis, and apoptosis of the noninfarcted myocardium. These beneficial effects of Prx-3 gene overexpression were associated with the attenuation in mitochondrial oxidative stress, mtDNA decline, and dysfunction (27). The specific localization of Prx-3 in the mitochondria suggests that mitochondrial oxidative stress plays an important role in the development and progression of heart failure and the antioxidant localized specifically within the mitochondria provides a primary line of defense against this disease process.

\section{Mitochondrial transcription factor A (TFAM)}

TFAM is a nucleus-encoded protein that binds mtDNA and promotes transcription of mtDNA. TFAM not only regulates mtDNA transcription and replication (28), but also maintains mtDNA copy number. In fact, Tfam knockout mice, which had a $50 \%$ reduction in their transcript and protein levels, exerted a $34 \%$ reduction in the mtDNA copy number, $22 \%$ reduction in the mitochondrial transcript levels, and partial reduction in the cytochrome $c$ oxidase levels in the heart (29). Moreover, cardiac-specific disruption in the Tfam gene in mice exhibited dilated cardiomyopathy in association with a reduced amount of mtDNA and mitochondrial transcripts (30). The transfection of antisense plasmids in culture, designed to reduce the expression of TFAM, effectively decreased the levels of mitochondrially encoded transcripts (31). On the contrary, the forced overexpression of TFAM could produce the opposite effect (32). These lines of evidence obtained from knockout mice have established a critical role for TFAM in regulation of mtDNA copy number and mitochondrial function as well as maintenance of the physiological function of the heart in vivo. In addition, a reduction in TFAM expression has been demonstrated in several forms of cardiac failure (15, 33-35).

By using transgenic mice that overexpress human TFAM gene, we examined whether TFAM could protect the heart from mtDNA deficiencies and attenuate LV remodeling and failure after MI (36). TFAM overexpression could ameliorate the decline in mtDNA copy number and preserve it at a normal level in post-MI hearts from TFAM transgenic mice. TFAM overexpression might increase the steady-state levels of mtDNA by directly stabilizing mtDNA. Consistent with alterations in mtDNA, the decrease in oxidative capacities seen in MI was also prevented. Moreover, TFAM played an important role in myocardial protection against remodeling and failure. Therefore, even though one should be cautious to extend the present results obtained from post-infarct heart failure model into other forms of heart failure due to hypertension, cardiomyopathy, or valvular heart disease, the activation of TFAM expression could ameliorate the pathophysiological processes seen in myocardial failure. However, the methods to activate TFAM especially in animal models of heart failure or patients have not yet been established.

Several factors may be attributable to the protective effects conferred by TFAM against myocardial remodeling and failure. First, TFAM overexpression prevented the decrease in mtDNA copy number and mitochondrial electron transport function, which may contribute to the decrease in myocardial oxidative stress. The decreased oxidative stress could contribute to the amelioration of cardiac hypertrophy, apoptosis, and interstitial fibrosis (36). A recent study by Ekstrand et al demonstrated that the overexpression of human TFAM in mice increases the mtDNA copy number (37). These lines of evidence imply the primary importance of TFAM as a regulatory mechanism of mtDNA copy number. TFAM has been shown to directly interact with mtDNA to form nucleoids $(38,39)$. Therefore, increased TFAM may increase the steady-state levels of mtDNA by directly binding and stabilizing mtDNA in transgenic mice. Second, TFAM overexpression may induce mitochondrial biogenesis, which, however, is thought to be unlikely because the number and size of the mitochondria assessed by electron microscopy were not altered.

\section{Conclusions}

To improve the prognosis of patients with heart failure, novel therapeutic strategies based on a novel insight into the pathophysiology of myocardial remodeling and failure need to be developed. The approach by regulating mitochondrial oxidative stress and DNA damage may contribute to the establishment of the effective treatment strategies for patients with heart failure. Oxidative stress is involved not only in heart failure, but also in various cardiovascular diseases including atherosclerosis, hypertension, and even aging. Therefore, therapeutic strategies to modulate this maladaptive response should definitely become a target for future 
extensive investigation and could have a broader application.

grants from the Ministry of Education, Science and Culture, Japan (No. 12670676, 14370230, 17390223, 17659223).

The work presented in this article was supported in part by

\section{References}

1. Ho KK, Pinsky JL, Kannel WB, Levy D. The epidemiology of heart failure: the Framingham Study. J Am Coll Cardiol 22: 6A-13 A, 1993.

2. McCord JM. Oxygen-derived free radicals in postischemic tissue injury. N Engl J Med 312: 159-163, 1985.

3. Belch JJ, Bridges AB, Scott N, Chopra M. Oxygen free radicals and congestive heart failure. Br Heart J 65: 245-248, 1991.

4. Mallat Z, Philip I, Lebret M, Chatel D, Maclouf J, Tedgui A. Elevated levels of 8-iso-prostaglandin F2alpha in pericardial fluid of patients with heart failure: a potential role for in vivo oxidant stress in ventricular dilatation and progression to heart failure. Circulation 97: 1536-1539, 1998.

5. Hill MF, Singal PK. Antioxidant and oxidative stress changes during heart failure subsequent to myocardial infarction in rats. Am J Pathol 148: 291-300, 1996.

6. Hill MF, Singal PK. Right and left myocardial antioxidant responses during heart failure subsequent to myocardial infarction. Circulation 96: 2414-2420, 1997.

7. Ide T, Tsutsui H, Kinugawa S, et al. Direct evidence for increased hydroxyl radicals originating from superoxide in the failing myocardium. Circ Res 86: 152-157, 2000.

8. Tsutsui H, Ide T, Hayashidani S, et al. Greater susceptibility of failing cardiac myocytes to oxygen free radical-mediated injury. Cardiovasc Res 49: 103-109, 2001.

9. Ide T, Tsutsui H, Kinugawa S, et al. Mitochondrial electron transport complex I is a potential source of oxygen free radicals in the failing myocardium. Circ Res 85: 357-363, 1999.

10. Sawyer DB, Colucci WS. Mitochondrial oxidative stress in heart failure: "oxygen wastage" revisited. Circ Res 86: 119-120, 2000.

11. Giulivi C, Boveris A, Cadenas E. Hydroxyl radical generation during mitochondrial electron transfer and the formation of 8hydroxydesoxyguanosine in mitochondrial DNA. Arch Biochem Biophys 316: 909-916, 1995.

12. Williams RS. Mitochondrial gene expression in mammalian striated muscle. Evidence that variation in gene dosage is the major regulatory event. J Biol Chem 261: 12390-12394, 1986.

13. Williams RS. Canaries in the coal mine: mitochondrial DNA and vascular injury from reactive oxygen species. Circ Res 86: 915916, 2000.

14. Ballinger SW, Patterson C, Yan CN, et al. Hydrogen peroxide- and peroxynitrite-induced mitochondrial DNA damage and dysfunction in vascular endothelial and smooth muscle cells. Circ Res 86: 960-966, 2000.

15. Ide T, Tsutsui H, Hayashidani $S$, et al. Mitochondrial DNA damage and dysfunction associated with oxidative stress in failing hearts after myocardial infarction. Circ Res 88: 529-535, 2001.

16. Siwik DA, Tzortzis JD, Pimental DR, et al. Inhibition of copperzinc superoxide dismutase induces cell growth, hypertrophic phenotype, and apoptosis in neonatal rat cardiac myocytes in vitro. Circ Res 85: 147-153, 1999.

17. Siwik DA, Pagano PJ, Colucci WS. Oxidative stress regulates collagen synthesis and matrix metalloproteinase activity in cardiac fibroblasts. Am J Physiol Cell Physiol 280: C53-C60, 2001.

18. Creemers EE, Cleutjens JP, Smits JF, Daemen MJ. Matrix metalloproteinase inhibition after myocardial infarction: a new approach to prevent heart failure? Circ Res 89: 201-210, 2001.

19. Spinale FG, Coker ML, Thomas CV, Walker JD, Mukherjee R, Hebbar L. Time-dependent changes in matrix metalloproteinase activity and expression during the progression of congestive heart failure: relation to ventricular and myocyte function. Circ Res 82: 482-495, 1998.

20. Rohde LE, Ducharme A, Arroyo LH, et al. Matrix metalloproteinase inhibition attenuates early left ventricular enlargement after experimental myocardial infarction in mice. Circulation 99: 30633070, 1999.

21. Hayashidani S, Tsutsui H, Ikeuchi M, et al. Targeted deletion of MMP-2 attenuates early LV rupture and late remodeling after experimental myocardial infarction. Am J Physiol Heart Circ Physiol 285: H1229-H1235, 2003.

22. Rajagopalan S, Meng XP, Ramasamy S, Harrison DG, Galis ZS. Reactive oxygen species produced by macrophage-derived foam cells regulate the activity of vascular matrix metalloproteinases in vitro. Implications for atherosclerotic plaque stability. J Clin Invest 98: 2572-2579, 1996.

23. Kinugawa $S$, Tsutsui $H$, Hayashidani $S$, et al. Treatment with dimethylthiourea prevents left ventricular remodeling and failure after experimental myocardial infarction in mice: role of oxidative stress. Circ Res 87: 392-398, 2000.

24. Le CT, Hollaar L, van der Valk EJ, van der Laarse A. Buthionine sulfoximine reduces the protective capacity of myocytes to withstand peroxide-derived free radical attack. J Mol Cell Cardiol 25: 519-528, 1993.

25. Toussaint O, Houbion A, Remacle J. Relationship between the critical level of oxidative stresses and the glutathione peroxidase activity. Toxicology 81: 89-101, 1993.

26. Shiomi T, Tsutsui $H$, Matsusaka $H$, et al. Overexpression of glutathione peroxidase prevents left ventricular remodeling and failure after myocardial infarction in mice. Circulation 109: 544-549, 2004.

27. Matsushima S, Ide T, Yamato M, et al. Overexpression of mitochondrial peroxiredoxin-3 prevents left ventricular remodeling and failure after myocardial infarction in mice. Circulation 113: 17791786, 2006.

28. Scarpulla RC. Nuclear activators and coactivators in mammalian mitochondrial biogenesis. Biochim Biophys Acta 1576: 1-14, 2002.

29. Larsson NG, Wang J, Wilhelmsson $\mathrm{H}$, et al. Mitochondrial transcription factor A is necessary for mtDNA maintenance and embryogenesis in mice. Nat Genet 18: 231-236, 1998.

30. Wang J, Wilhelmsson H, Graff C, et al. Dilated cardiomyopathy and atrioventricular conduction blocks induced by heart-specific inactivation of mitochondrial DNA gene expression. Nat Genet 21: 133-137, 1999.

31. Inagaki H, Kitano $S$, Lin KH, Maeda $S$, Saito T. Inhibition of mitochondrial gene expression by antisense RNA of mitochondrial transcription factor A (mtTFA). Biochem Mol Biol Int 45: 567573, 1998.

32. Montoya J, Perez-Martos A, Garstka HL, Wiesner RJ. Regulation of mitochondrial transcription by mitochondrial transcription factor A. Mol Cell Biochem 174: 227-230, 1997.

33. Lebrecht D, Setzer B, Ketelsen UP, Haberstroh J, Walker UA. Time-dependent and tissue-specific accumulation of mtDNA and respiratory chain defects in chronic doxorubicin cardiomyopathy. Circulation 108: 2423-2429, 2003.

34. Kanazawa A, Nishio Y, Kashiwagi A, Inagaki H, Kikkawa R, Horiike K. Reduced activity of mtTFA decreases the transcription in mitochondria isolated from diabetic rat heart. Am J Physiol Endocrinol Metab 282: E778-E785, 2002. 
35. Garnier A, Fortin D, Delomenie C, Momken I, Veksler V, VenturaClapier R. Depressed mitochondrial transcription factors and oxidative capacity in rat failing cardiac and skeletal muscles. J Physiol 551: 491-501, 2003.

36. Ikeuchi M, Matsusaka H, Kang D, et al. Overexpression of mitochondrial transcription factor a ameliorates mitochondrial deficiencies and cardiac failure after myocardial infarction. Circulation 112: 683-690, 2005.
37. Ekstrand MI, Falkenberg M, Rantanen A, et al. Mitochondrial transcription factor A regulates mtDNA copy number in mammals. Hum Mol Genet 13: 935-944, 2004.

38. Alam TI, Kanki T, Muta $T$, et al. Human mitochondrial DNA is packaged with TFAM. Nucleic Acids Res 31: 1640-1645, 2003.

39. Takamatsu $C$, Umeda $S$, Ohsato $T$, et al. Regulation of mitochondrial D-loops by transcription factor A and single-stranded DNAbinding protein. EMBO Rep 3: 451-456, 2002.

(C) 2006 The Japanese Society of Internal Medicine http://www.naika.or.jp/imindex.html 